Kirdoda, J. , Dumas, D. C.S., Millar, R. W. , Mirza, M. M. , Paul, D. J. , Kuzmenko, K., Vines, P., Greener, Z. and Buller, G. S. (2020) Geiger Mode Ge-on-Si Single-Photon Avalanche Diode Detectors. In: 2019 IEEE 2nd British and Irish Conference on Optics and Photonics (BICOP), London, UK, 11-13 Dec 2020, ISBN

9781728149486 (doi:10.1109/BICOP48819.2019.9059574)

There may be differences between this version and the published version. You are advised to consult the publisher's version if you wish to cite from it.

\title{
http://eprints.gla.ac.uk/214037/
}

Deposited on: 16 April 2020

Enlighten - Research publications by members of the University of Glasgow http://eprints.gla.ac.uk 


\section{Geiger mode Ge-on-Si Single-Photon Avalanche Diode Detectors}

\author{
Jaroslaw Kirdoda, Derek C.S. Dumas, Ross W. Millar, \\ Muhammad M. Mirza, Douglas J. Paul \\ School of Engineering \\ University of Glasgow \\ Glasgow, United Kingdom \\ Douglas.Paul@glasgow.ac.uk
}

\author{
Kateryna Kuzmenko, Peter Vines, Zoë Greener, \\ Gerald S. Buller \\ Insitute of Photonics and QT \\ Heriot-Watt Univesity \\ Edinburgh, United Kingdom \\ G.S.Buller@hw.ac.uk
}

\begin{abstract}
High efficiency single photon avalanche detectors (SPADs) based on the Ge-on-Si material system are a promising emerging technology for detection in the SWIR region. Here we demonstrate record level single photon detection efficiencies of $38 \%$ at $1310 \mathrm{~nm}$ with an operation temperature of $125 \mathrm{~K}$. This was achieved using a novel planar geometry which allowed us to achieve an NEPs of $3 \times 10^{-16} \mathrm{WHz}^{-}$ $1 / 2$ and a reduced afterpulsing when compared to $\mathrm{InGaAs} / \mathrm{InP}$ based SPADs operated in nominally identical conditions.
\end{abstract}

Keywords - single-photon avalanche diode detector, Germanium on Silicon, single-photon counting, Geiger mode

\section{INTRODUCTION}

Over the last decade there has been a growing demand, in both the military and civilian sectors, for single photon detectors capable of high detection efficiencies and low dark count rates[1],[2]. This interest was expressed explicitly by the UK government, which announced $£ 270$ million investment in 2013 to span five year in a National Quantum Technologies Programme that would help to accelerate both development and implementation of quantum technologies in both private and military sector applications. With uses in time of flight ranging LIDAR technologies[6],[8], quantum key distribution [9], remote gas sensing and applications in quantum communication[10], various single-photon detection techniques have been developed. Commercially available Si SPAD devices are a popular choice for many applications due to their relatively low cost and near room temperature operation. Due to the Si bandgap however, these devices are limited to operation below $1000 \mathrm{~nm}$. This leaves market's demand for a low cost technology that would be robust, eye safe and sensitive in the short-wave infrared region (SWIR) unsatiated. Wavelengths between $1310 \mathrm{~nm}$ and $1550 \mathrm{~nm}$ are especially interesting for the quantum communications and especially range-finding. The lower photon energy at $1550 \mathrm{~nm}$ laser compared to the $850 \mathrm{~nm}$ laser could enable a twenty-fold increase in laser source power while still remaining eye safe [11]. Being able to use lasers running at wavelengths close to $1550 \mathrm{~nm}$ for range-finding, allows for increased penetration length in the atmosphere (compared to near-visible) while taking into account environmental conditions such as smoke, fog, snow, rain, snow, and haze [3], [4]. An additional important factor that needs to be taken into consideration is the influence of solar radiation, which acts as an additional source of noise in the LIDAR measurement. The influence of this noise, however, is also significantly reduced at wavelengths around $1550 \mathrm{~nm}$ [7]. So far, the only commercially available devices capable of such operation are either based on the superconducting nanowires or are SPAD structure based on InGaAs/InP. These devices are not appropriate for mass-market applications, however, since they are expensive to manufacture and, in the case of nanowire devices, require cryogenic temperatures for operation. As an alternative to these technologies we present a high efficiency SPAD based on the Ge-on-Si material system. Due to the narrower bandgap of $\mathrm{Ge}$ than $\mathrm{Si}$, it can be used as an absorber to allow for extended absorption, potentially up to $\sim 1600 \mathrm{~nm}$. Furthermore, the high material quality of Si avalanche layers leads to reduced trap states, and therefore reduced afterpulsing compared to III-V devices. [12], [13]. Additionally using Si as a substrate allows for an easier integration with existing CMOS production lines that would allow for significant reduction in production costs of the devices. Such detectors could be adapted for realizing LIDAR systems for autonomous vehicles, where the ability to perform long range range-finding through atmospheric obscurants could prove crucial in adapting these technologies for mass market [5], [6]. Previous Ge-on-Si SPADs have used a mesa etch to define the device area; here, we present pseudo-planar devices which led to a three order of magnitude improvement in dark count rates (DCR) per unit area as well as single photon detection efficiency (SPDE) increased by an order of magnitude, with operation up to $175 \mathrm{~K}$.

\section{FABRICATION AND CHARACHTERISATION}

The wafers used for these devices were $150 \mathrm{~mm}$ diameter $\mathrm{n}^{++}-\mathrm{Si}(001)$ with a $1.5 \mu \mathrm{m}$ thick i-Si multiplication region epitaxially grown on top. This was then followed by a boron implantation of $\mathrm{p}^{-} \mathrm{Si}$ charge sheet that regulates the electric

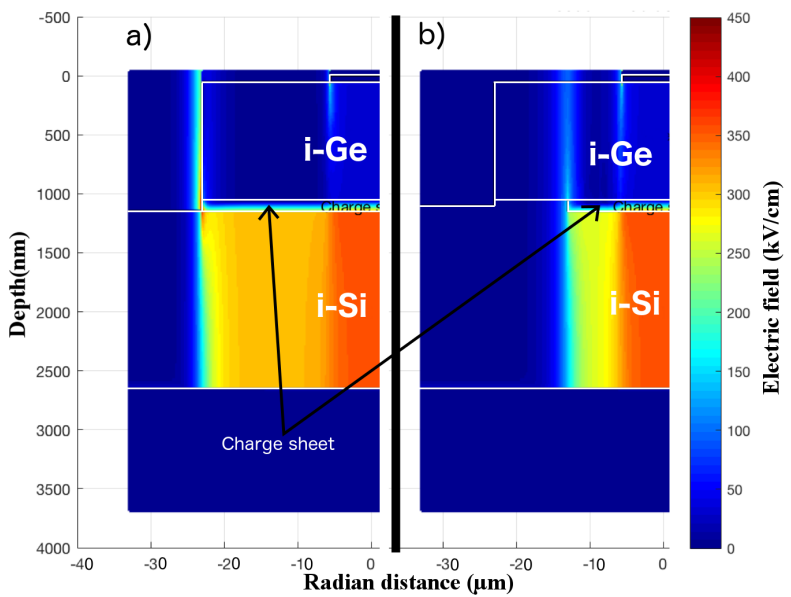

Fig. 1. Electric field simulation for a cross section through a Ge-on-Si SPAD device for previous Mesa and current Planar design. A high electric field is present at the side wall of a mesa type device a) while for a planar device b) electric field is confined within the device. 
field between the absorption and multiplication layers. The Implanted ions were then activated with an anneal using a rapid thermal anneal tool. Subsequently, a $1 \mu \mathrm{m}$ thick i-Ge absorption layer was epitaxially grown and capped with a heavily doped $\mathrm{p}^{++}$-Ge top contact layer. In the design presented in previous work cylindrical mesa etch was used to define an active area of the device [13]. current leakage

Mesa devices are known to have a larger interaction with generation-recombination centres on etched sidewalls, which can increase the DCR. Furthermore, as shown in Fig. 1a), the etch leads to a local 'hot-spots' of the electric field in both the $\mathrm{Si}$ and Ge, which can cause early breakdown of the device. To address this problem a new planar device design was investigated. In this design the charge sheet is selectively doped (rather than uniformly across the wafer). As can be seen in the Fig. 1b) this causes the electric field to be confined within the active area limited by the dimensions of the charge sheet. After initial investigation it was discovered that due to the non-zero background doping of the Ge layer there was current leakage between the neighbouring devices. An isolation trench etch was introduced in the planar design to provide lateral insulation between the devices [15]. It was also discovered that the quality of the side wall of the insulation trench had a small effect on the shape of the current-voltage characteristic of the device suggesting that contrary to the simulation results there is a slight electric field present at the sample side wall in the planar devices. Differences between these two designs can be observed in current - voltage characteristic in Fig. 2. It can be seen that the planar device is exhibiting much sharper breakdown which is indicative of a high efficiency device.

The inset to Fig. 2 shows the current-voltage characteristics for a $100 \mu \mathrm{m}$ diameter planar device in dark conditions (black curve) and whilst illuminated with a $1310 \mathrm{~nm}$ laser (red curve). In the dark conditions the current before breakdown is extremely low, below $1 \mathrm{nA}$. In the case of the current-voltage characteristic under illumination with a $1310 \mathrm{~nm}$ laser, a photo-current can be observed, particularly above $15 \mathrm{~V}$. This is due to an effect called punch-through which indicates that electric field extends into Ge absorber layer causing carriers photogenerated by $1310 \mathrm{~nm}$

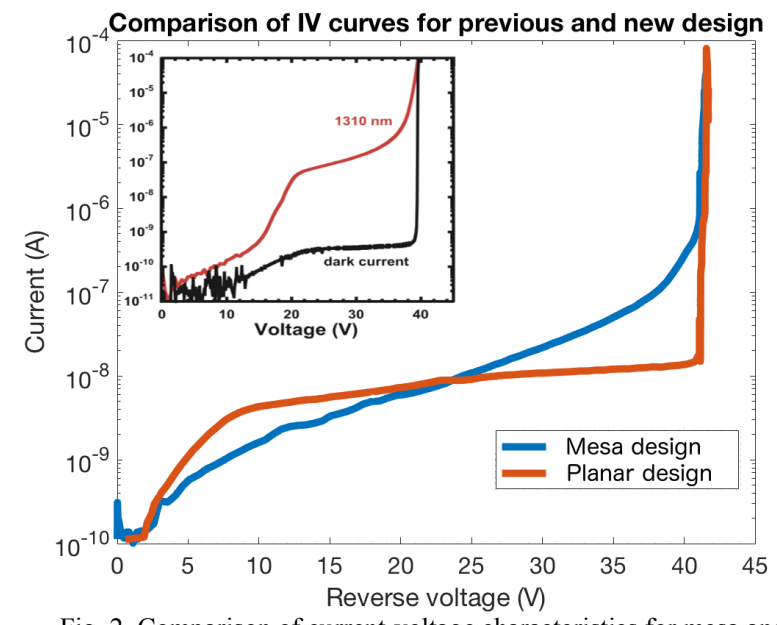

Fig. 2. Comparison of current-voltage characteristics for mesa and planar type $100 \mu \mathrm{m}$ diameter device at $100 \mathrm{~K}$. Inset : The current-voltage characteristic for a planar device at $78 \mathrm{~K}$ in dark and illuminated by a $1310 \mathrm{~nm}$ laser. illumination to be swept into the Si multiplication region and being registered as an increase in the current.

Single photon characterisation of the devices was carried out with a time corelated single photon counting technique (TCPSP) with a setup presented in Fig. 3. After initial characterisation the devices were mounted on a cold finger of a cryostat allowing for measurement with a precise temperature control between $77 \mathrm{~K}$ and $300 \mathrm{~K}$. Pulsed lasers were used to illuminate the device, one with a set operational wavelength of $1310 \mathrm{~nm}$, and a tunable laser for wavelengths between $1310 \mathrm{~nm}$ and $1550 \mathrm{~nm}$. Half of the beam was directed into the power meter, allowing for a continuous output power monitoring and the other half was directed towards the calibrated programable attenuator set to ensure an average of less than 0.1 photons per pulse. This is to ensure that the chance of getting more than one photon in a pulse would be negligible ensuring that we are operating in the single photon regime. This is a commonly accepted technique for performing single photon measurements [13], [15]. For the measurements the devices were kept at a voltage just below the breakdown. Prior to the expected time of photon arrival onto the device, a regulated electric pulse was being superimposed onto the applied bias. This added pulse brings the device above the breakdown voltage with the additional voltage above breakdown known as the excess bias. This allows the avalanche event to be triggered either by a carrier generated by an absorbed photon or by a carrier generated due to other processes like thermal generation or trap state depopulation (dark count). The timing of the incoming photon was precisely controlled by a master clock connected both to the laser and gate generator. The electrical output generated by the device was then measured by a Edinburgh Instrument TCC900 photon counting card. Devices continue to avalanche up until the end of the gate pulse (which in this case was set to 50ns), after which the removal of the excess bias brings the device back below the breakdown voltage quenching the breakdown. A TCSPC card then generates histograms for both light and dark conditions. From these measurements it is possible to extract the single photon detection efficiency (SPDE) (see Fig. 4) and dark count rates (DCR) (see Fig. 5). The timing jitter of these devices is defined as the full width half maximum (FWHM) of the histogram under illumination,

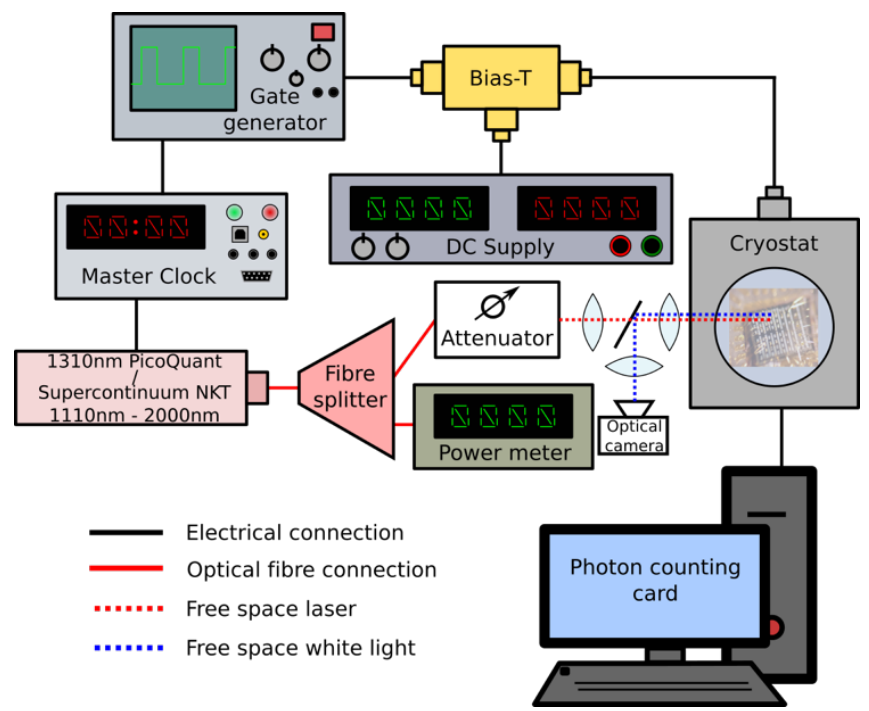

Fig. 3. A diagram of a single photon characterisation setup used to perform Single Photon Detection Efficiency, Dark Count Rate and jitter measurements. 


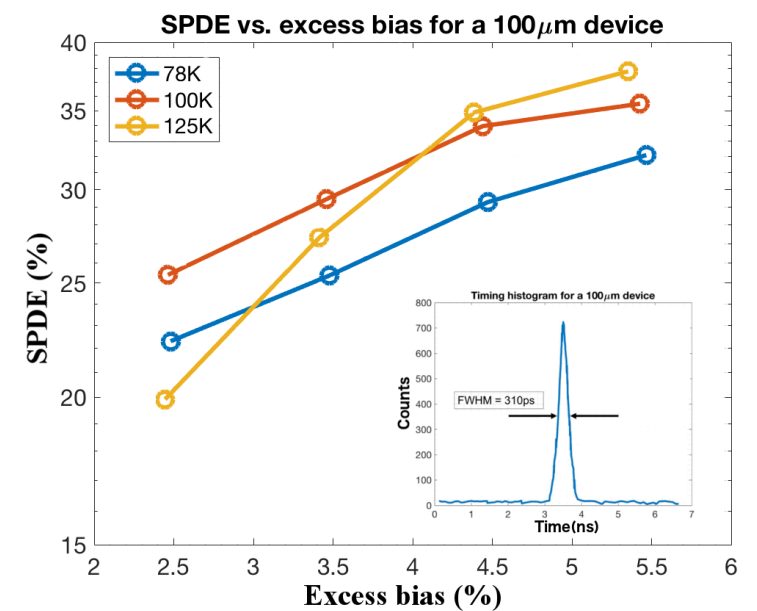

Fig. 4. The SPDE as a function of excess bias for a $100 \mu \mathrm{m}$ diameter device at $77 \mathrm{~K}, 100 \mathrm{~K}$ and $125 \mathrm{~K}$. Inset: timing histogram for a $100 \mu \mathrm{m}$ diameter device with a 310 ps FWHM.

and was measured to be 310 ps as presented in the inset of Fig. 4.

The SPDE as a function of excess bias for a $100 \mu \mathrm{m}$ diameter planar device is presented in Fig. 4. The value of the SPDE increases with increasing excess bias and increasing temperature reaching a record value at $125 \mathrm{~K}$ for a Ge-on-Si type device of $38 \%$ [12 - 14]. The ability to perform SPDE measurements is however limited by the DCR that increases with an increasing excess bias or increasing temperature which ultimately at the highest values masks any single photon counts. This limits the high temperature operation. For SWIR devices, operation on a Peltier cooler is desirable to minimise the cost of the system but also to allow longer wavelength operation. This is caused by a shift of a direct absorption band in Ge towards shorter wavelengths at lower temperatures as confirmed by simulated values of the $\mathrm{Ge}$ absorption coefficient for various temperatures (Fig. 6). The simulations suggest that the direct band absorption at $1550 \mathrm{~nm}$ could be achieved at $245 \mathrm{~K}$. One approach to increase the temperature of operation is to decrease the diameter of the SPAD. A device with a smaller diameter should have lower DCR which would allow for single photon detection at higher temperatures. With increasing temperature of operation direct absorption band for Ge shifts to longer wavelengths allowing

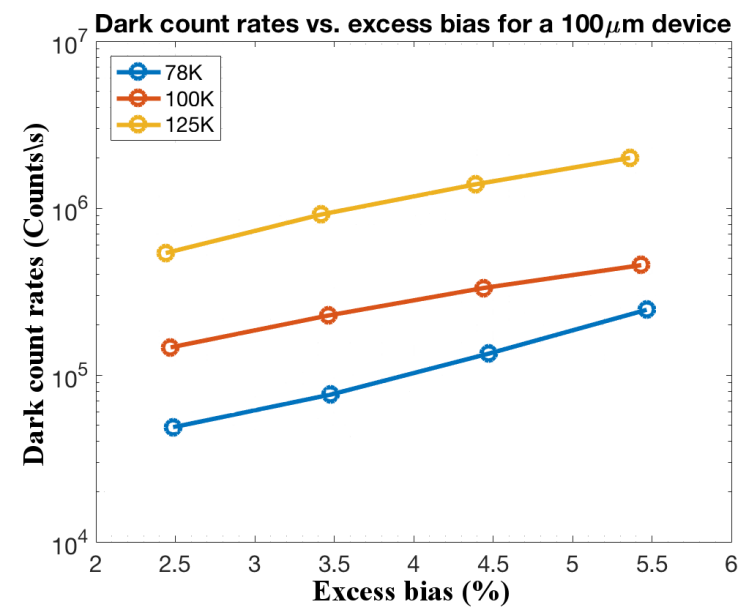

Fig. 5. The DCR as a function of excess bias for a $100 \mu \mathrm{m}$ diameter device at $77 \mathrm{~K}, 100 \mathrm{~K}$ and $125 \mathrm{~K}$.

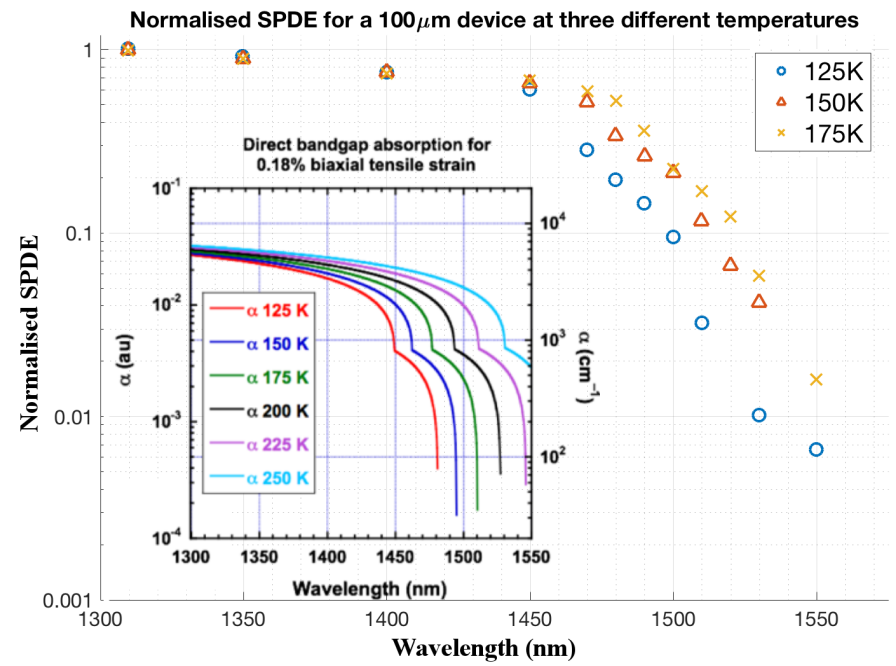

Fig. 6. Normalised SPDE as a function of wavelength for different temperature. Inset: Calculated values of Ge absorption coefficient as a function of wavelength.

for more photons to be absorbed therefore making single photon detection possible at $1550 \mathrm{~nm}$ wavelength.

Afterpulsing is an effect related to the trapping centers in the crystalline lattice created by unwanted deep level, point defects, dislocations or dangling bonds at surfaces As the avalanche event is triggered by either an incoming photon or a dark count a large amount of carriers is released inside the avalanche region. Some of these carriers can be trapped any of defects, deep level impurities or dislocations. After the device is brought below the breakdown voltage these trapping centers start to release trapped carriers. If the device would be brought above the breakdown voltage before all of the trapped carriers depopulate, such a released carrier could trigger another avalanching event causing a false positive detection of a single photon. Most of these trapping centers however, naturally depopulate over time. With this afterpulsing can be defined as a measure of the probability of a dark count occurring due to release of a carrier that was trapped in a previous avalanche event. Therefore the introduction of a longer wait between the gate of the device between two detection times would significantly reduce the probability of an afterpulse occurring. This would mean however that the device cannot be operated at high frequency if an increase in false positives is to be avoided. Afterpulsing is heavily influenced by the quality of the multiplication layer used in the SPAD device. In Ge-on-Si SPADs the multiplication occurs in $\mathrm{Si}$ which can be grown with a very few trapping centers. This puts Ge-on-Si SPADs in an advantageous position compared to devices based on InGaAs/InP for which InP is used as a multiplication layer, which has a much higher defect density and therefore higher afterpulsing [17 - 19].

To gain a better understanding of the afterpulsing in a Ge-onSi SPAD, a measurement was carried out on a $100 \mu \mathrm{m}$ diameter device. This was later compared to a commercially available $26 \mu \mathrm{m}$ diameter InGaAs/InP device under nominally identical conditions which consisted of conducting the measurement at a temperature of $125 \mathrm{~K}$ under the illumination of a $1310 \mathrm{~nm}$ laser while setting the SPDE to $17 \%$. The results of this investigation are presented in Fig. 9. It can be seen that in the case of shorter delays between the gates, the rate of afterpulsing for a $100 \mu \mathrm{m}$ diameter Ge-on-Si SPAD is five times lower than a $26 \mu \mathrm{m}$ diameter $\mathrm{InGaAs} / \mathrm{InP}$ device. This 




Fig. 7.The afterpulsing probability as a function of gate length for a $100 \mu \mathrm{m}$ diameter Ge-on-Si SPAD compared to a $26 \mu \mathrm{m}$ diameter InGaAs/InP under nominally the same conditions.

suggest that Ge-on-Si SPAD devices could be more viable for applications where high repetition rates are crucial.

\section{CONCLUSION}

We have presented a planar $100 \mu \mathrm{m}$ diameter Ge-on-Si SPAD device which achieved record breaking 38\% SPDE at $125 \mathrm{~K}$ with DCR per unit area three orders of magnitude lower than previously reported Ge-on-Si SPADs [12], [13]. The FWHM recorded for a single photon detection event was recorded to be $310 \mathrm{ps}$. The noise equivalent power was measured to be $3 \times 10^{-16} \mathrm{WHz}^{1 / 2}$. Five times lower afterpulsing rate have been measured while comparing afterpulsing between our $100 \mu \mathrm{m}$ device and a $26 \mu \mathrm{m}$ InGaAs/InP SPAD under nominally identical conditions.

\section{ACKNOWLEDGMENT}

This work was supported by DSTLX- 1000092774 and UK EPSRC projects EP/N003446/1, EP/K015338/1, EP/L024020/1, EP/M01326X/1 and EPN003225/1.

\section{REFERENCES}

[1] R.H. Hadfield, "Single-photon detectors for optical quantum information applications" Nat. Photon. vol. 3, pp. 696-705, 2009.

[2] G.S. Buller and R.J. Collins, "Single-photon generation and detection" Meas. Sci. Technol. vol. 21, 012002, 2010.

[3] F. Christnacher, S. Schertzer, N. Metzger, E. Bacher, M. Laurenzis, and R. Habermacher, "Influence of gating and of the gate shape on the penetration capacity of range-gated active imaging in scattering environments" Opt. Exp. Vol. 23, pp. 32897-32908, 2015.
[4] A. Arnulf, Bricard A., Cure J., and C. Veret, "Transmission by haze and fog in the spectral region 0.35 to 10 microns", J. Opt. Soc. Am. Vol. 47, pp. 491-498, 1957.

[5] R. Tobin et al., "Three-dimensional single-photon imaging through obscurants" Opt. Express, vol. 27 , pp. 4590-4611, 2019.

[6] A.M. Pawlikowska, A. Halimi, R.A. Lamb and G.S. Buller, "Singlephoton 3D imaging at up to $10 \mathrm{~km}$ range" Opt. Exp. vol. 25, pp. 11919$11931,2017$.

[7] R. E. Bird, R. L. Hulstrom, and L. J. Lewis, "Terrestrial solar spectral data sets", Solar Energy, vol 30, pp. 563-573, 1983.

[8] R. Tobin, A. Halimi, A. McCarthy, X. Ren, K. J. McEwan, S. McLaughlin, and G. S. Buller, "Long-range depth profiling of camouflaged targets using single-photon detection" Opt. Eng., Vol 57(3), pp. 031303, 2017.

[9] G. S. Buller, R. E. Warburton, S. Pellegrini, J. S. Ng, J. P. R. David, L. J. J. Tan, A. B. Krysa, and S. Cova, "Single-photon avalanche diode detectors for quantum key distribution" IET Optoelec., vol 1, pp. 249254, 2007.

[10] S. Cova, M. Ghioni, F. Zappa, A. Tosi, I. Rech, A. Gulinatti, and S. Tisa, "Single-photon avalanche detectors for quantum communications", Conf. Optical Fiber Comm. (OFC/NFOEC),Nat. Fiber Optic Eng. Conf., pp. 1-3, 2010.

[11] “IEC-60825-1:2014," tech. rep., IEC, 2014.

[12] R. E. Warburton et al., "Ge-on-Si single-photon avalanche diode detectors: design, modeling, fabrication, and characterization at wavelengths 1310 and $1550 \mathrm{~nm}$ ", IEEE Trans. Electron Dev., vol. 60, no. 11, pp. 3807-3813, 2013.

[13] A.Y. Loudon et al., "Enhancement of the infrared Detection Efficiency in Si Photon Counting Avalanche Photodiode using SiGe Absorbing Layers" Optics Letters, vol. 27, pp. 219-221, 2002.

[14] P. Vines, K. Kuzmenko, J. Kirdoda, D.C.S. Dumas, M.M. Mirza, R.W. Millar, D.J. Paul and G.S. Buller, "High performance planar Ge-on-Si single-photon avalanche diode detectors" Nature Comms. vol. 10, 1086, 2019.

[15] N.J.D. Martinez et al., "Single photon detection in a waveguidecoupled Ge-on-Si lateral avalanche photodiode" Opt. Exp. vol. 25, pp. 16130- 16139, 2017.

[16] S. Pellegrini, R. E. Warburton, L. J. . Tan, Jo Shien Ng, A. B. Krysa, K. Groom, J. P.. David, S. Cova, M. J. Robertson, and G. S. Buller, "Design and performance of an InGaAs-InP single-photon avalanche diode detector", IEEE J. Quantum Electron., vol. 42(4), pp. 397-403, 2006.

[17] G.S Buller, S. Pellegrini, R.E. Warburton, J.S. Ng, L.J.J. Tan, A. Krysa, J.P.R. David, and S. Cova, "Semiconductor avalanche diode detectors for quantum cryptography", IEEE LEOS Newsletter, vol. 20, 2006.

[18] X. Jiang, M. A. Itzler, R. Ben-Michael, and K. Slomkowski, "InGaAsP-InP avalanche photodiodes for single photon detection", IEEE J. Sel. Top. Quantum Electron., vol. 13, pp. 895-905, 2007.

[19] B. Korzh, T. Lunghi, K. Kuzmenko, G. Boso, and H. Zbinden, „Afterpulsing studies of low-noise InGaAs/InP single-photon negativefeedback avalanche diodes", J. Modern Optics, vol. 62, pp. 1151-1157, 2015 . 